\title{
Preface
}

\author{
Carles Gomez $^{\mathrm{a}}$, Andrea Prati ${ }^{\mathrm{b}}$, Hamid Aghajan $^{\mathrm{c}, \mathrm{d}}$ and Juan Carlos Augusto ${ }^{\mathrm{e}}$ \\ ${ }^{a}$ Wireless Networks Group, Department of Network Engineering, Universitat Politecnica de Catalunya, Spain \\ ${ }^{\mathrm{b}}$ Department of Information Engineering, University of Parma, Italy \\ c imec, IPI, Gent University, Belgium \\ ${ }^{\mathrm{d}}$ Ambient Intelligence Research (AIR) Lab, Stanford University, USA \\ e Department of Computer Science and Research Group on Development of Intelligent Environments, Middlesex \\ University, $U K$
}

\section{This issue}

This issue of JAISE is composed of eight papers. The review process for these papers was supervised by our editors Stefano Chessa, Jinshi Cui, Carles Gomez, Hans Guesgen, Alex Mihailidis, Andrés Muñoz Ortega, Andrea Prati, Caifeng Shan, Liping Shen, and Tong Zhen, whom we thank for their service. The back pages of this issue include naming and acknowledging the contributing reviewers of the manuscripts submitted to JAISE in 2016.

Many energy efficiency policies rely on adjusting the demand-supply balance at the peak hours using a dynamic pricing structure. The effectiveness of these policies depends on the flexibility of device operation selectivity by the users, which is reliant on the exchange of price information between the grid and its consumers. The paper "An Open Solution to Provide Personalized Feedback for Building Energy Management" by Monacchi et al. proposes an interactive energy management system to timely inform users on energy usage and promote an optimal usage. Data from households in Italy and Austria is used to gain insight into usage behavior and drive the design of more effective systems. The paper proposes an open platform in which various feedback concepts can be implemented and evaluated.

Sedentary activity has the potential to be a behavioral predictor of functional decline in older adults. Such behavior has a number of important implications on the health of the elderly, such as increasing the risk of disability and falls, or leading to other medical complications, or it may be a sign of conditions such as depression or cognitive decline. The paper "Exploratory Analysis of Older Adults' Sedentary Be- havior in the Primary Living Area Using Kinect Depth Data" by Banerjee et al. describes case studies of clinically significant changes in sedentary behavior of older adults. The proposed algorithms use a Kinect sensor to detect postural changes from and to the sitting posture.

Estimation of the walking patterns of an elderly person can yield important information about the physical or cognitive health of the person. Walking speed is one of the main attributes of many gait analysis approaches. The paper "Estimation of Human Walking Speed by Doppler Radar for Elderly Care" by Rui et al. proposes the use of a Doppler radar for inhouse estimation of a user's walking pace. The paper examines the received signal in the time domain and produces speed estimates through identifying its zerocrossing locations. The system has been tested via experiments in senior residences.

Monitoring of the heart rate in an in-home environment provides significant benefit for elder care. Unobtrusive and continuous monitoring of vital signs such as heart rate has wide applications at homes and hospitals. The paper "Heart Rate Monitoring Using Hydraulic Bed Sensor Ballistocardiogram" by Rosales et al. proposes a hydraulic bed sensor which captures the superposition of the heart rate and respiration signals. The paper reports on two algorithmic methods in the time and frequency domains to extract the heart rate from the captured signal.

Most probabilistic models employed in activity recognition approaches require labeled datasets for adapting themselves to different users and environments. This requirement poses a major problem for the scalability of automated human activity recognition systems. The paper "Active Learning with Un- 
certainty Sampling for Large Scale Activity Recognition in Smart Homes" by Alemdar et al. proposes an active learning method based on uncertainty sampling. The paper aims to reduce the annotation effort by selecting only the most informative data points for annotation. An additional benefit of the proposed approach is that the learned system can be redeployed in new environments and serve to better initialize the relevant parameters.

Labeling children's social play behavior is an important process in children's peer-play analysis which is traditionally done by experienced professionals. With the growing volume of data, automatic methods for labeling are increasingly required. The paper "Computer vision analysis for children's social play classification in peer-play scenarios" by Duan et al. presents a method to classify children's social play behavior in peer-play scenarios into the three categories of solitary play, parallel play and group play. Two vision-based cues of proximity and head orientation are used as the classification features. The method has been tested on a dataset of children's peer-play scenarios collected by psychology researchers.

Security issues of wireless sensor networks which are deployed in inaccessible terrain need to be handled with energy efficient approaches. The limited resources present in the sensor nodes as well as the communication constraints make the energy efficiency parameters the most important factors in the design of these networks. The objective of any intrusion detection framework is hence defined as designing robust mechanisms capable of handling attacks in an energy efficient manner. The paper "A Survey on Energy Efficient Intrusion Detection in Wireless Sensor Networks" by Ghosal and Halder introduces the major topics of energy efficient intrusion detection in wireless sensor networks, and covers topics such as the fundamentals of intrusion detection techniques, as well as the various energy saving mechanisms used in different architectural models. The paper mentions the earlier achievements in energy efficient intrusion detection, and discusses the existing problems facing the researchers of this field.
Localization is one of the main functions for many smart environment services. Most of the newest applications need to know the user's location to customize their services, monitor people, or track Internet-ofThings objects. Moreover, the location can also be used for detecting the user's activities and to provide services based on them. Accurate positioning is also considered as a fundamental enabling technology for future 5G mobile networks. Many different technologies and indoor positioning systems have been proposed to deal with location indoors. The paper "A Realistic Evaluation of Indoor Positioning Systems Based on Wi-Fi Fingerprinting: The 2015 EvAAL-ETRI Competition” by Torres-Sospedra et al. presents results from comparing different Wi-Fi fingerprinting algorithms on the same dataset. The algorithms where implemented by different teams during the EvAAL-ETRI indoor localization competition, which was held at the Sixth International Conference on Indoor Positioning and Indoor Navigation in 2015.

\section{Upcoming issues}

The following is the list of upcoming issues of JAISE:

- May 2017: Thematic Issue on Intelligent Systems, Applications and Environments for the Industry of the Future.

- July 2017: Regular Issue.

- September 2017: Thematic Issue on Humancentred AmI: Cognitive Approaches, Reasoning and Learning.

- November 2017: Regular Issue.

- January 2018: Thematic Issue based on papers from "Intelligent Environments 2017".

More information on the call for papers to the future thematic issues is available on the webpage of JAISE at: http://www.iospress.nl/journal/journal-of-ambientintelligence-and-smart-environments/. 